\title{
Evaluación del efecto de prensado en prótesis totales maxilares sobre la movilidad de los dientes artificiales, en función del tipo de inclusión
}

\section{Evaluation of the effect of full denture pressing on the artificial teeth mobility, in function of two type of flasking}

\section{Gennari Filho $H^{*}$, Coelho Goiato $M^{*}$, Mara Negrão Alves L**, Ramos Silva C**, Mary Falcón Antenucci R**}

\section{RESUMEN}

El presente trabajo evaluó el movimiento de los dientes artificiales en prótesis totales maxilares, sometidas a diversas presiones durante el prensado y el efecto de este en relación al material usado en la inclusión. Treinta y seis réplicas de prótesis totales previamente confeccionadas, fueran divididas en seis grupos: Los grupos G1, G2 y G3 fueron incluidos con yeso tipo III, a una presión de 800, 1.000 y 1.250 kgf respectivamente y los grupos S1, S2 y S3 fueron incluidos con una barrera de silicona y yeso, a una presión de 800, 1.000 y 1.250 $\mathrm{kgf}$, respectivamente. Las prótesis fueron medidas en puntos demarcados previamente en los segundos molares, premolares e incisivo central con el programa de computación gráfica (AutoCad 2000), después de cada etapa: encerado, polimerización y emuflado.

La comparación entre estas dos fases permitió observar que: en los grupos (G1 y S1) donde se utilizó la menor presión (800 kgf) ocurrieron las mayores alteraciones en el posicionamiento de los dientes artificiales y que el grupo que mostró menores alteraciones fue cuando se utilizó $1.250 \mathrm{kgf}$ de presión y la inclusión fue con yeso.

Palabras clave: Dientes artificiales, resinas acrílicas, prótesis totales superiores.

\section{SUMMARY}

The present study evaluated artificial teeth movement in complete dentures, submitted to different flasking loading and the effect of these loading pressures on the material used in the flasking procedure. Thirty-six dentures were fabricated and equally divided into six groups: Groups G1, G2 and G3 were processed with type III gypsum and 800, 1000 and 1250 kgf loading pressures, respectively, and groups S1, S2 and S3 were processed with silicone barrier and 800,1000 and $1250 \mathrm{kgf}$ loading pressures, respectively. In order to evaluate the artificial teeth movement, measurements were performed from previously determined points down in the second molars tips and in the second pre-molars cusps and in the central incisor. Those points were scanned (Scan Jet 6100C - Hewlett Packard) and then the measurements were performed and analyzed through the use of the AutoCad 2000 software, during the wax and the post-polymerization phases.

The comparison between these two phases allowed the following observations: in groups (G1 and S1) where less loading pressure was used ( $800 \mathrm{kgf}$ ), the movements in the artificial teeth positions were the highest, and the group that presented the lowest changes was the one processed with type III gypsum and $1250 \mathrm{kgf}$ loading pressure.

* Profesores del Departamento de Materiales Odontológicos y Prótesis.

** Alumnas del curso de maestría en Odontología. Área de Prótesis Dental. 
Key words: Artificial teeth, acrylic resins, upper complete denture.

Fecha de recepción: Enero 2008.

Aceptado para publicación: Marzo 2008.

Gennari Filho H, Coelho Goiato M, Mara Negrão Alves L, Ramos Silva C, Mary Falcón Antenucci R. Evaluación del efecto de prensado en prótesis totales maxilares sobre la movilidad de los dientes artificiales, en función del tipo de inclusión. Av. Odontoestomatol 2008; 24 (5): 323-332.

\section{INTRODUCCIÓN}

Las prótesis totales, en función de la inestabilidad de los materiales empleados en su confección, sufren alteraciones en sus dimensiones, que si no son detectadas a tiempo, pueden, provocar lesiones en los tejidos blandos.

La modificación de la posición dental, en función de estas alteraciones dimensionales, genera contactos oclusales indeseables que, bajo ciertas circunstancias, provocan más que lesiones comunes, incomodidad al paciente.

De este modo, la búsqueda de la alteración "cero", ha sido motivo de ciertas investigaciones, con la intención de observar lo que se puede hacer para reducir al mínimo tal situación.

Con ese fin, algunos autores como Tucker \& Freeman (1), Maineri et al. (2), Cury (3), Zannetti (4), Shibayama (5), Gennari Filho et al. $(6,7)$ y Shibayama (8), estudiaron la forma de inclusión, es decir, el empleo de diferentes materiales de inclusión como: Yeso tipo III y Silicona utilizada como una barrera sobre los dientes o solamente yeso.

En 2005, Gennari Filho et al. (9) en un estudio comparativo sobre diferentes formas de inclusión, comparó la inclusión de prótesis totales superiores con yeso y con una barrera de silicona, con revestimiento total y parcial. Concluyendo que la inclusión con revestimiento total de silicona no mostró una alteración significativa de la posición relativa de los dientes.

Otros investigadores como Kimura et al (10), Levin et al. (11), Sanders et al. (12), Goiato et al. (13), Gen- nari Filho et al. $(6,7)$ estudiaron la forma de polimerización de la resina acrílica: En agua en ebullición o por medio del microondas; demostrando que la polimerización por medio del microondas mostró una tendencia muy fuerte a la alteración de la posición de los dientes artificiales.

Más allá de estos aspectos, muchos otros estudios fueron realizados con la intención de analizar el movimiento de los dientes artificiales en relación a los materiales usados (Goiato et al. 14); en lo referente al tipo de pulimento, Goiato et al. (15); relacionado con el espesor de las bases de prueba, Mazaro (16); la interrelación entre el movimiento dental y la profundidad del paladar, Vedovatto (17) y la eficacia de las siliconas para la inclusión de prótesis totales, Assunção et al. (18). Por medio de estos estudios, muchos parámetros fueron establecidos y se observó, que considerables mejoras fueron alcanzadas en base a los resultados obtenidos.

Aunque, desde el punto de vista estadístico, esas alteraciones son insignificantes; estas tienen un efecto clínico nocivo, comprobando que un buen ajuste oclusal es esencial para lograr el confort deseado.

Otro aspecto que llama la atención, esta relacionado con los factores que producen estas alteraciones, como la cantidad de presión que se debe ejercer en la mufla durante la inclusión de la resina acrílica. Russi et al. (19) hicieron un estudio donde se observó la influencia del tipo de prensa con relación a las alteraciones que ocurrieron en las muflas. Dando por resultado, la alteración de la dimensión vertical de oclusión. Más recientemente, otros autores como Cunha et al. (20) proponen el uso de muflas "bimaxilares HH" con la intención de reducir al mínimo las 
Gennari Filho H, Coelho Goiato M, Mara Negrão Alves L, Ramos Silva C, Mary Falcón Antenucci R. Evaluación del efecto de prensado en prótesis totales maxilares sobre la movilidad de los dientes artificiales,

alteraciones que ocurren, en función de la inclusión en conjunto de las prótesis total superior e inferior, esto es corroborado por Tenguan et al. (21) que afirmaron, que no había necesidad de hacer remontaje de las prótesis en articulador, para el ajuste oclusal, sino apenas pequeños ajustes directamente en la boca.

\section{MATERIALES Y MÉTODO}

En un modelo edéntulo superior fueron enfilados los dientes artificiales (2D- 32M - Vipi Plus - VIPI Dental Ltda.) con la ayuda de un articulador semiajustable, teniendo como modelo antagonista el modelo inferior correspondiente. La prótesis total superior, aún en cera, fue moldada con la silicona, permitiendo su reproducción con los dientes posicionados, en sus respectivos lugares (Figura 1). A seguir, se realizó el vaciado de cera rosa $n^{\circ} 7$ fundida (Figura 2), y se adaptó el modelo previamente confeccionado (Figura 3). Después de la solidificación de la cera, la prótesis (diente + cera + modelo) fue retirada.

Esta técnica permitió la reproducción de 36 prótesis totales superiores enceradas de forma idéntica (Figura 4), que fueran divididas en 6 grupos de seis réplicas cada uno:

- Grupo G1: Inclusión en yeso tipo III, a 800 kgf. de presión.

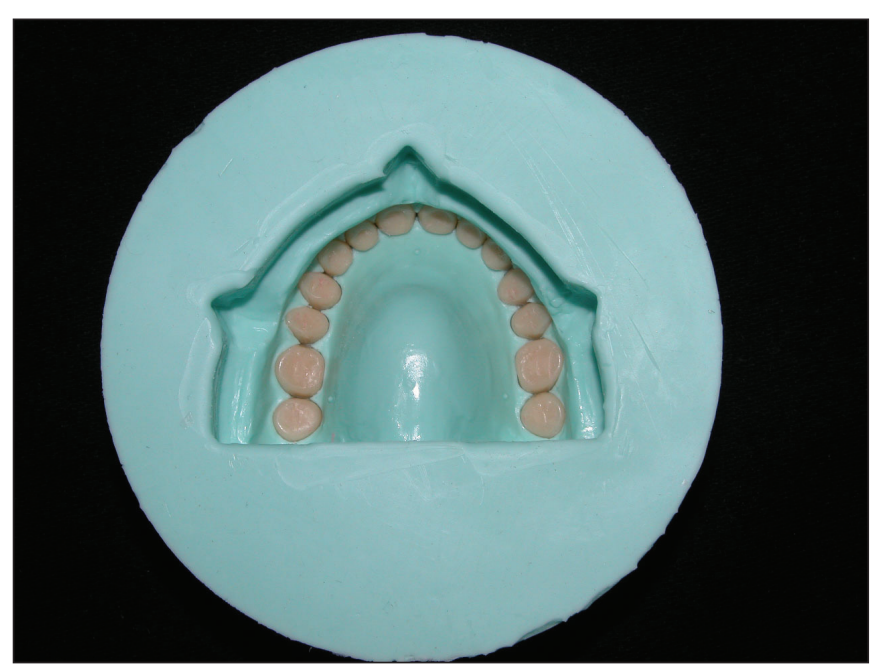

Fig 1. Dientes posicionados en sus respectivos lugares.

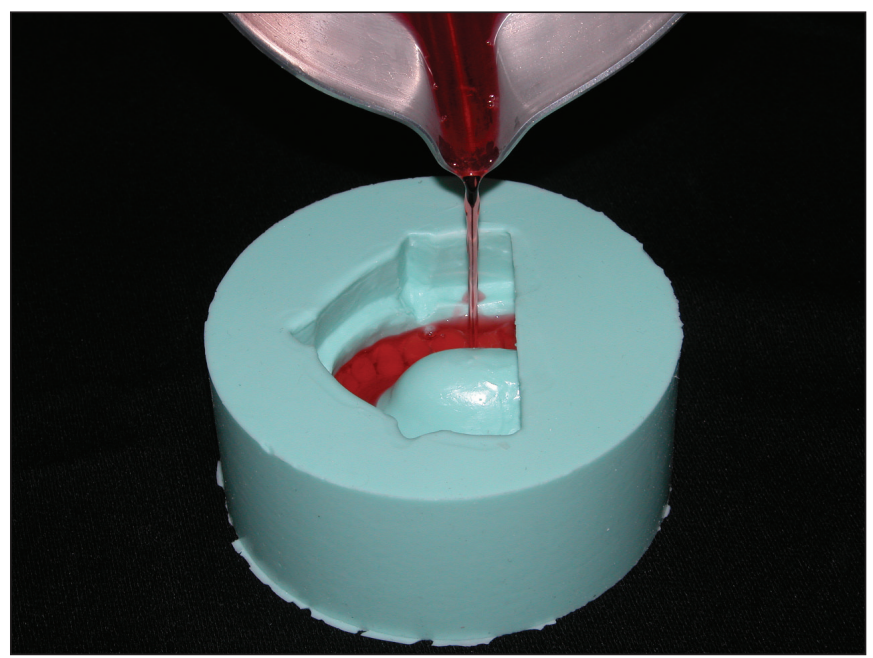

Fig 2. Vaciado de cera rosa $n^{\circ} 7$

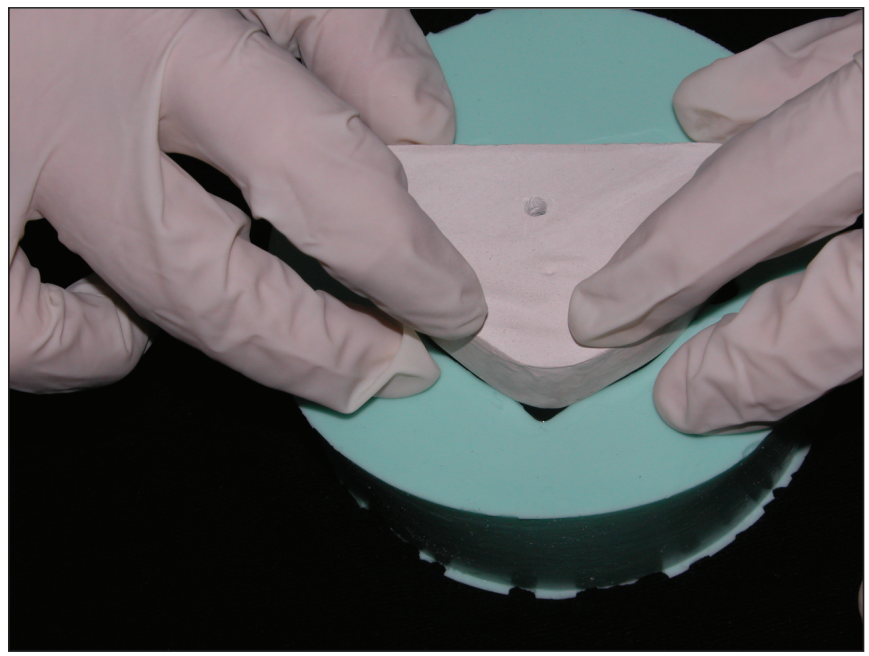

Fig 3. Adaptación del modelo previamente confeccionado.

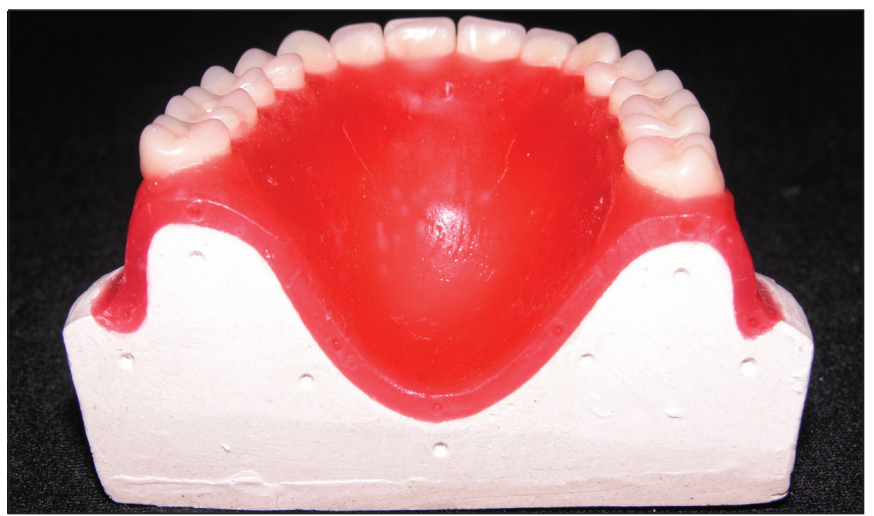

Fig 4. Reproducción de las replicas de forma idéntica. 
— Grupo G2: Inclusión en yeso tipo III, a 1.000 kgf de presión.

- Grupo G3: Inclusión en yeso tipo III, a 1.250 kgf de presión.

- Grupo S1: Inclusión en silicona/yeso tipo III, a 800 kgf de presión.

- Grupo S2: Inclusión en silicona/yeso tipo III, a $1.000 \mathrm{kgf}$ de presión.

- Grupo S3: Inclusión en silicona/yeso tipo III, a 1.250 kgf de presión.

El número de las muestras (6) fueron suficientes, en función que, el método ya fue comprobado con el coeficiente de correlación de Pearson, en trabajos previos, Vedovatto (17).

En la inclusión con yeso, toda la base de la prótesis, incluyendo los dientes fueron cubiertos con yeso tipo III y en la inclusión con silicona, la base de la prótesis y los dientes fueron cubiertos con este material (Figura 5) y el restante con yeso tipo III.

Después de la eliminación de la cera, las muflas fueron abiertas para la eliminación del exceso de humedad y para el resfriamiento completo, antes de la ejecución de los pasos siguientes.

La resina acrílica utilizada para el procedimiento de inclusión fue Vipi Wave (Dental Vipi Ltda.), apropiada para microondas, proporcionada y manipulada

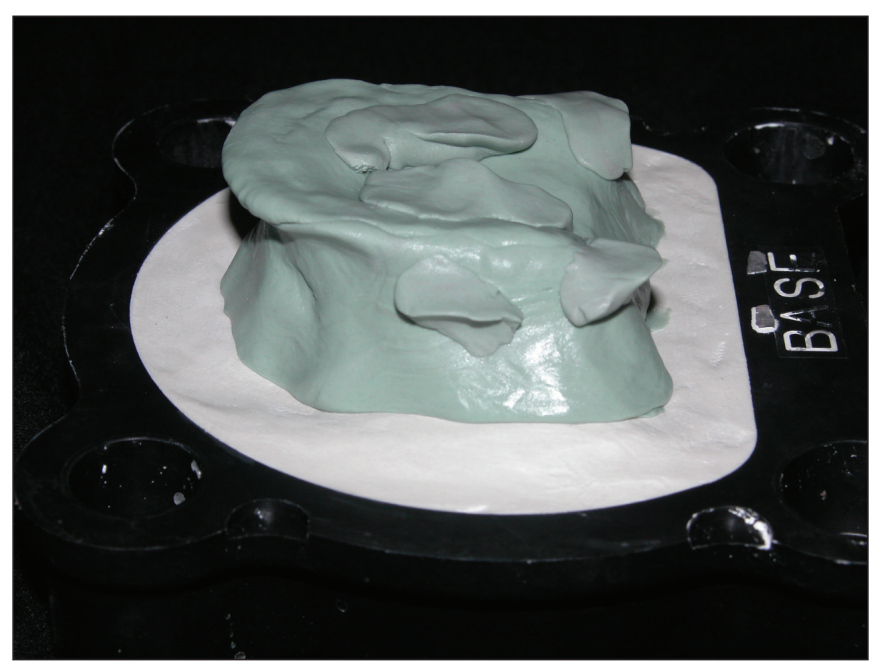

Fig 5. Inclusión con barrera de silicona. de acuerdo con las especificaciones del fabricante. Al alcanzar la fase plástica, fue colocada en la mufla y presionada en la prensa hidráulica, lenta y progresivamente hasta alcanzar la presión media de cada grupo, manteniéndola a presión constante por 5 minutos:

- Grupos G1 y S1: 800 kgf.

- Grupos G2 y S2: 1.000 kgf.

- Grupos G3 y S3: 1.250 kgf.

El tiempo de polimerización de bancada fue de 1 hora, Gennari Filho et al. (7), y el ciclo de polimerización llevó 20 minutos a $20 \%$ de potencia (800 watts) y 5 minutos a $60 \%$ de potencia, con enfriamiento a temperatura ambiente por un período de 24 horas. Inmediatamente después del emuflado, los modelos fueron separados de las prótesis con ayuda de un cincel neumático (Renfert 5022 - Alemania) para prevenir posibles fracturas, limpiados luego con agua y cepillo.

Las medidas fueron tomadas en dos momentos (Figuras 6 y 7$)$ :

a) Prótesis en cera.

b) Después de la polimerización.

De modo que estos puntos fueron repetidos de igual manera en todas las réplicas fue construida una guía

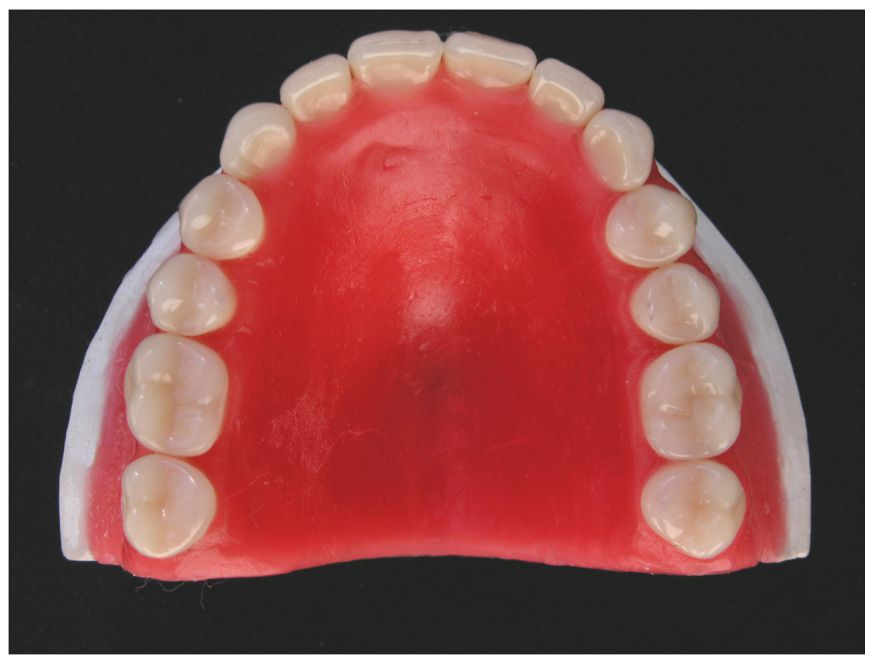

Fig 6. Momento de la tomada de medida en cera. 
Gennari Filho H, Coelho Goiato M, Mara Negrão Alves L, Ramos Silva C, Mary Falcón Antenucci R. Evaluación del efecto de prensado en prótesis totales maxilares sobre la movilidad de los dientes artificiales, en función del tipo de inclusión

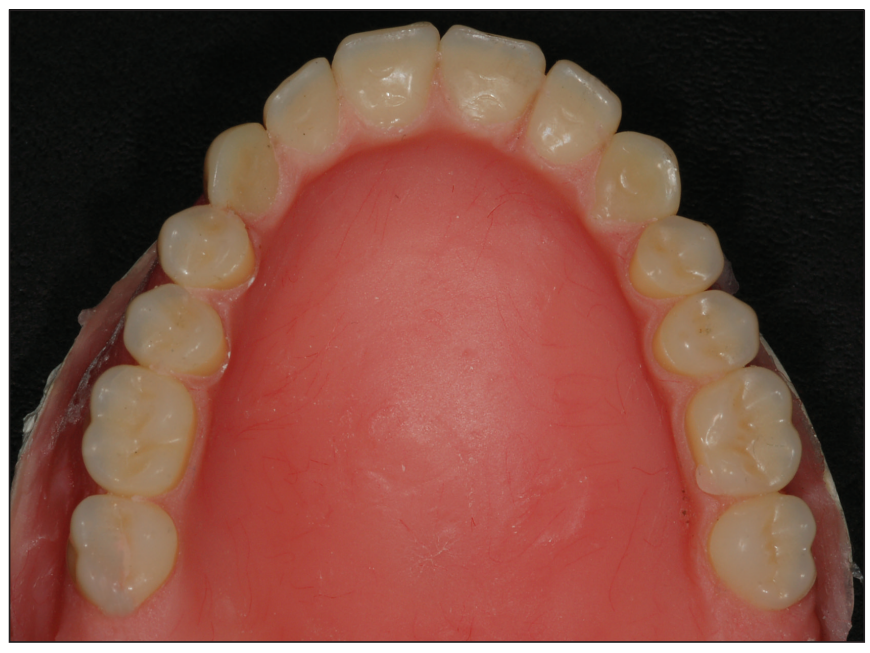

Fig 7. Momento de la tomada de medida después de la polimerización.

de resina acrílica incolora sobre las superficies dentales de la prótesis seleccionada, perforada en los puntos demarcados, lo que permitió reproducirlos en las otras 35 prótesis (Figura 8). Los puntos marcados fueron cubiertos con grafito para tornarse aún más evidentes (Figura 9). Para conseguir las medidas entre los puntos; las prótesis en cera fueron digitalizadas (Scan jet 6100C - Hewlett-Packard) junto con un bloque metálico de $1 \times 1 \mathrm{~cm}$ permitiendo que el programa fije los verdaderos valores, favoreciendo el redimensionamiento de los modelos. Posteriormente a la digitalización de cada prótesis, (Fi-

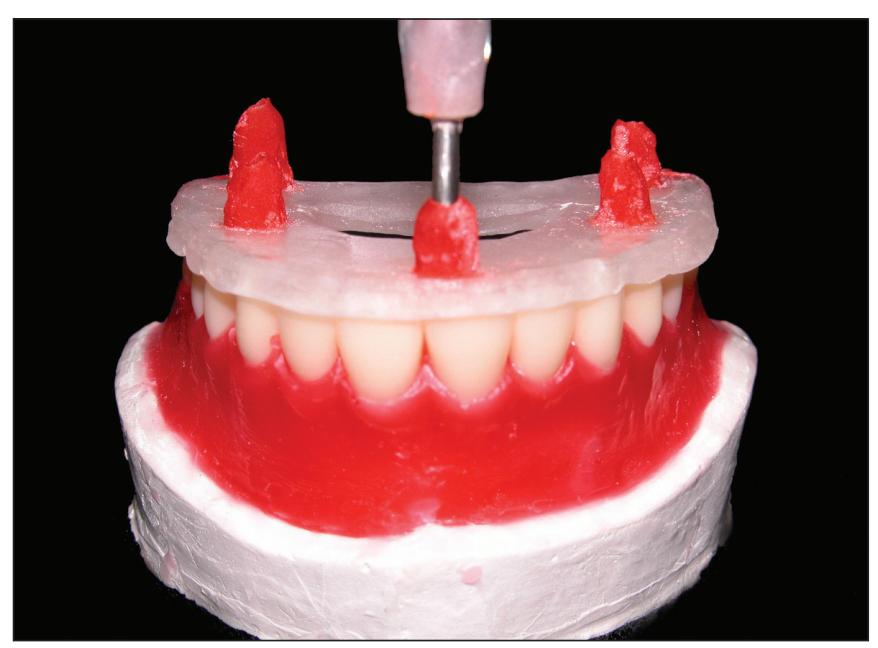

Fig 8. Demarcación con la guía y el estilete.

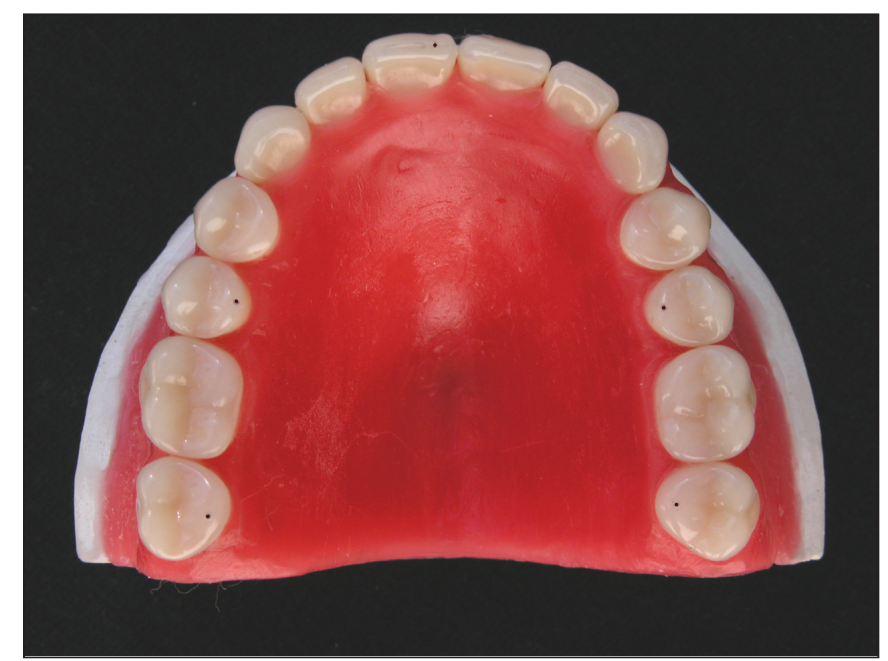

Fig 9. Puntos evidenciados con el grafito.

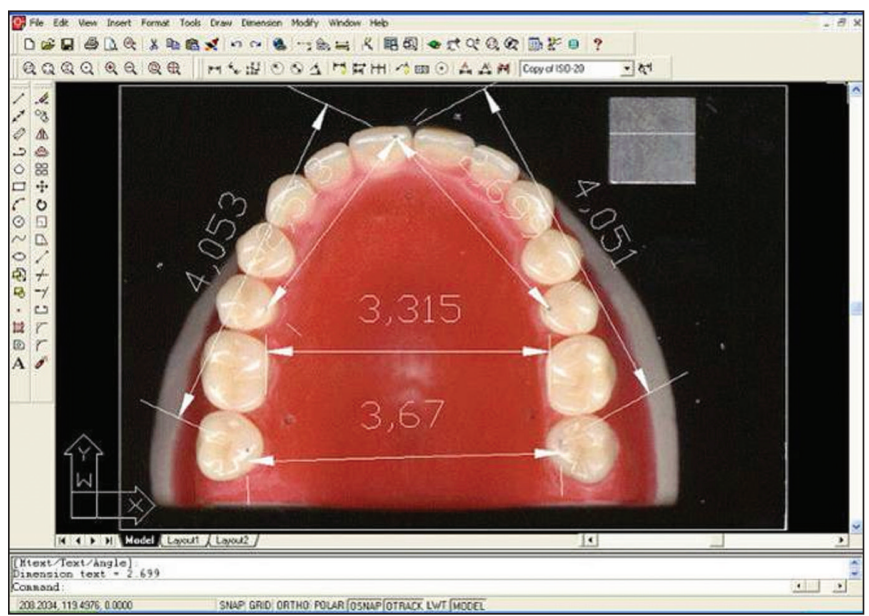

Fig 10. Imagen exportada al programa de computación gráfica AutoCad.

gura 10), las imágenes fueron exportadas al programa AutoCad 2000, para medir las distancias entre los puntos (Figura 11), dando valores a los segmentos. Después de la polimerización, toda la operación fue repetida.

\section{RESULTADO Y DISCUSIÓN}

La tabla 1, muestra los valores promedios de las medidas de todos los segmentos de las réplicas en la fase de encerado y en la fase de polimerización de los grupos incluidos con yeso, siendo el grupo G1, 


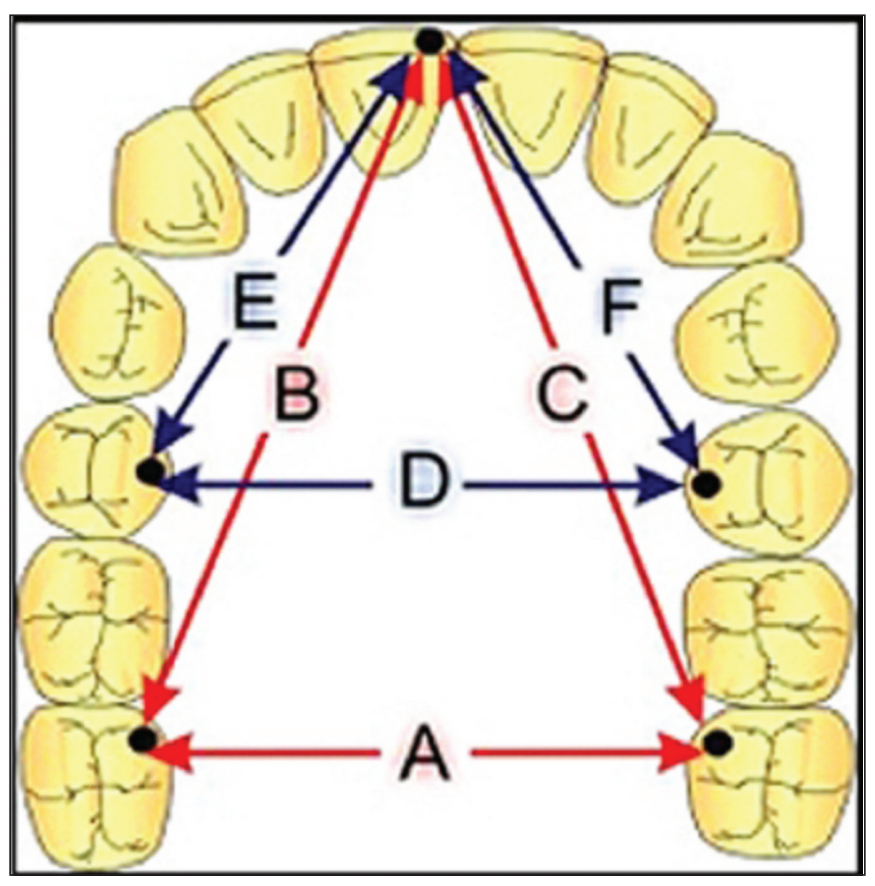

Fig 11. Puntos y segmentos que se medirán.

en el cual fue utilizado la presión media de $800 \mathrm{kgf}$, Grupo G2, presión de 1.000 kgf y grupo G3, presión de 1.250 kgf.

En la tabla 2 se muestran los valores promedios de las medidas de los segmentos de las réplicas de los grupos S1, S2 y S3, que fueron incluidos con la barrera de silicona con las presiones de 800, 1.000 y $1.250 \mathrm{kgf}$, respectivamente.

Puede ser evidenciado que, independientemente del tipo de barrera y de la presión usada; ocurrieron alteraciones en las posiciones de los dientes artificiales. Esto puede ser comprobado, por la diferencia de los valores promedios, debido a que la base de resina acrílica sufrió diversos tipos de tensiones durante su confección, dando por resultado distorsiones, con magnitud variable, motivando los movimientos dentales, lo que esta de acuerdo con los trabajos de algunos autores como Domiti (22) y Shibayama (5).

Este movimiento, como el descrito por Mazaro (16), es de difícil control por los múltiples factores que actúan en conjunto, como: las características intrínsecas de los materiales utilizados, contracción de polimerización, pérdida y absorción de agua, métodos de polimerización, influencia de la forma del paladar, y el proceso del emuflado, que fueron también observados por Shibayama et al. (8).

Al analizar los resultados de las tablas 1 y 2 se evidencia que, generalmente, el movimiento de dientes adopta un comportamiento de contracción linear en el sentido de disminuir la distancia entre los puntos de referencia, representados por la diferencia entre los valores promedios de los segmentos antes y

\begin{tabular}{|c|c|c|c|c|c|c|c|c|c|c|c|}
\hline & \multicolumn{11}{|c|}{$\begin{array}{l}\text { TABLA 1.- VALORES PROMEDIOS DE LAS MEDIDAS DE LOS SEGMENTOS DE LOS } \\
\text { GRUPOS INCLUIDOS CON YESO, BAJO PRESIÓN DE: } 800 \mathrm{KG} \text { (G1), } 1.000 \mathrm{KG} \text { (G2) } \\
\text { Y } 1.250 \mathrm{KG}(\mathrm{G} 3)\end{array}$} \\
\hline & \multicolumn{3}{|c|}{ Grupo G1 } & \multicolumn{4}{|c|}{ Grupo G2 } & \multicolumn{4}{|c|}{ Grupo G3 } \\
\hline & Cera & Poli & Dif. & & Cera & Poli & Dif. & & Cera & Poli & Dif. \\
\hline 1 & 3,362 & 3,356 & 0,005 & 1 & 3,374 & 3,351 & 0,023 & 1 & 3,355 & 3,357 & 0,003 \\
\hline 2 & 3,371 & 3,364 & 0,008 & 2 & 3,373 & 3,364 & 0,009 & 2 & 3,368 & 3,370 & 0,001 \\
\hline 3 & 3,362 & 3,327 & 0,035 & 3 & 3,371 & 3,362 & 0,009 & 3 & 3,360 & 3,364 & 0,005 \\
\hline 4 & 3,358 & 3,343 & 0,015 & 4 & 3,350 & 3,372 & 0,022 & 4 & 3,370 & 3,359 & 0,011 \\
\hline 5 & 3,362 & 3,351 & 0,011 & 5 & 3,365 & 3,364 & 0,001 & 5 & 3,367 & 3,368 & 0,001 \\
\hline \multirow[t]{2}{*}{6} & 3,346 & 3,294 & 0,052 & 6 & 3,356 & 3,346 & 0,010 & 6 & 3,375 & 3,357 & 0,017 \\
\hline & & & 0,021 & & & & 0,012 & & & & 0,006 \\
\hline
\end{tabular}




\begin{tabular}{|c|c|c|c|c|c|c|c|c|c|c|c|}
\hline & \multicolumn{11}{|c|}{$\begin{array}{c}\text { TABLA 2.- VALORES PROMEDIOS DE LAS MEDIDAS DE LOS SEGMENTOS DE LOS } \\
\text { GRUPOS INCLUIDOS CON SILICONA, BAJO PRESIÓN DE: } 800 \mathrm{KG}(\mathrm{S} 1), 1.000 \mathrm{KG} \text { (S2) } \\
\text { Y } 1.250 \mathrm{KG}(\mathrm{S} 3)\end{array}$} \\
\hline & \multicolumn{3}{|c|}{ Grupo S1 } & \multicolumn{4}{|c|}{ Grupo S2 } & \multicolumn{4}{|c|}{ Grupo S3 } \\
\hline & Cera & Poli & Dif. & & Cera & Poli & Dif. & & Cera & Poli & Dif. \\
\hline 1 & 3,370 & 3,346 & 0,025 & 1 & 3,383 & 3,374 & 0,009 & 1 & 3,353 & 3,367 & 0,014 \\
\hline 2 & 3,370 & 3,345 & 0,025 & 2 & 3,373 & 3,384 & 0,011 & 2 & 3,346 & 3,359 & 0,014 \\
\hline 3 & 3,369 & 3,352 & 0,016 & 3 & 3,376 & 3,387 & 0,011 & 3 & 3,360 & 3,358 & 0,002 \\
\hline 4 & 3,365 & 3,357 & 0,008 & 4 & 3,376 & 3,356 & 0,020 & 4 & 3,362 & 3,351 & 0,011 \\
\hline 5 & 3,372 & 3,309 & 0,063 & 5 & 3,376 & 3,376 & 0,000 & 5 & 3,359 & 3,357 & 0,002 \\
\hline \multirow[t]{2}{*}{6} & 3,368 & 3,342 & 0,026 & 6 & 3,392 & 3,374 & 0,018 & 6 & 3,367 & 3,353 & 0,015 \\
\hline & & & 0,027 & & & & 0,011 & & & & 0,009 \\
\hline
\end{tabular}

después del procesamiento de las prótesis, concordando con los trabajos de Goiato et al. (13), Gennari Filho et al. $(6,7)$ y Shibayama (5). Si analizamos los valores promedios de las diferencias de los grupos, independientemente de la señal $(-0+)$ que caracteriza las alteraciones de una forma general, la comprensión es más razonable, según lo evidenciado en los gráficos 1 y 2, referente a los diversos tipos de inclusión.

En la comparación entre los gráficos 1 y 2 , es posible observar que existen diferencias numéricas entre los resultados encontrados en ellos, tanto con la barrera de silicona (gráfico 2, grupos S1, S2 y S3) como con el yeso (gráfico 1, grupos G1, G2 y G3). Las mayores alteraciones en el posicionamiento de los dientes artificiales sucedieron en los grupos que sufrieron la acción de presión reducida (800kg) durante el prensado de la resina acrílica, es decir, Grupos G1 (yeso: 0,021 cm), y S1 (silicona: 0,027 $\mathrm{cm})$. Con el aumento de la presión el movimiento de los dientes artificiales disminuyó, grupo G2 $(0,0123)$ en relación al G3 $(0,0063)$ y grupo S2 $(0,0115)$ en relación al S3 $(0,0096)$. Probablemente, la mayor alteración en los grupos de presión reducida tiene relación con el mayor volumen de la resina acrílica que permanece en el interior de la mufla, generando un calentamiento más grande de la masa que podría promover el aumento de la tensión y la consecuente distorsión. Jamani y Moligoda (23) investigando el efecto del espesor de la prótesis en el movimiento dental durante el procesamiento de la misma, descubrieron un aumento en la distancia molar/molar en prótesis con base espesa, mientras que, prótesis

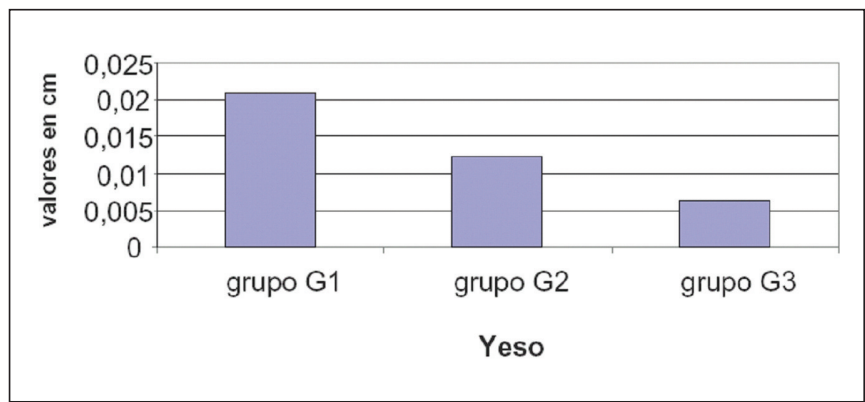

Gráfico 1. Alteraciones dimensionales de los tres grupos incluidos con yeso independiente de la contración o expansión.

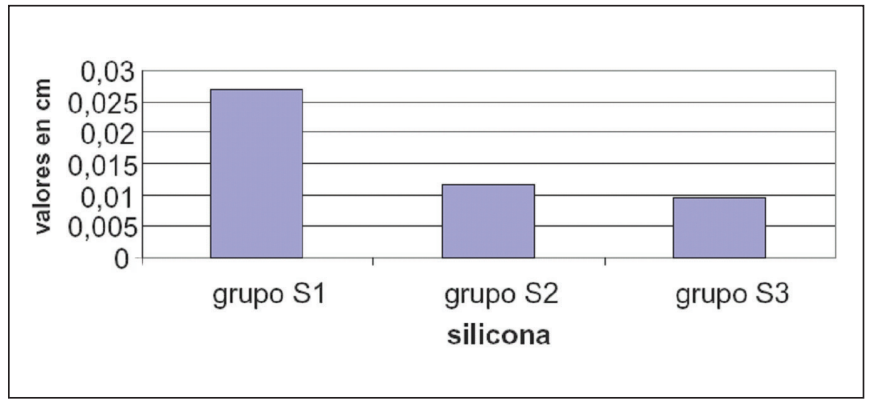

Gráfico 2. Alteraciones dimensionales de los tres grupos incluidos com silicona independiente de la contración o expansión. 


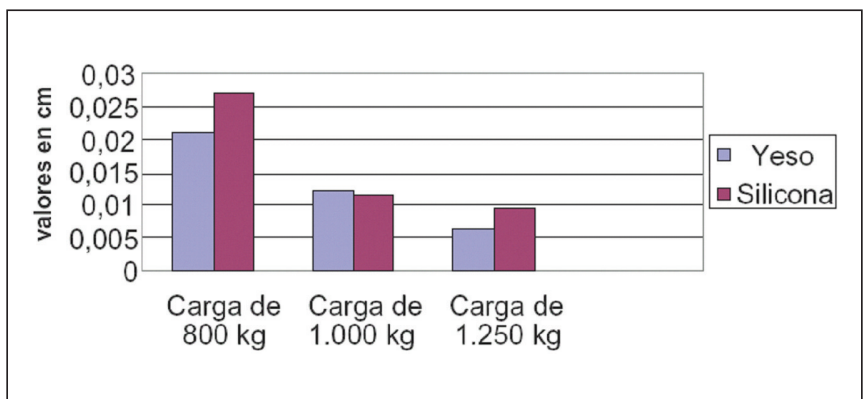

Gráfico 3. Comparación del efecto de las cargas en relación a las alteraciones en función del material de inclusion.

con base fina revelaron una pequeña reducción en la distancia molar/molar. Sadamori et al (24) relataron que prótesis de base gruesa presentaron características físicas inferiores cuando se compararon con muestras más delgadas, en función del ciclo de polimerización. Cuando se compara el tipo de material usado en la barrera (gráfico 3), con presión de 800 kgf, el grupo que presentó el menor movimiento fue el grupo donde se utilizó yeso tipo III (grupo G1), aunque la diferencia fue solamente 0,006 centímetros; esto se debe probablemente, a la menor resistencia de la silicona que se utilizó en el grupo (Grupo S1), permitiendo una mayor alteración en el posicionamiento dental durante la polimerización del acrílico. En la presión de 1.000 kgf, los dos materiales presentaron un valor promedio muy cercano, con diferencia solamente de $0,0008 \mathrm{~cm}, \mathrm{y}$ cuando se utilizó la presión de 1.250 kgf el material que mejor se comportó, comparando los valores promedios, fue el grupo de inclusión con yeso (grupo G3), con diferencia de 0,0033 centímetros. Debe ser considerado también que, según Zanetti (4), la inclusión de las prótesis con la técnica de barrera de silicona, es muy sensible a la alteración de la posición de los dientes, debido a la presión digital que se hace cuando se coloca la barrera, sobre la prótesis encerada.

Aunque las alteraciones dimensionales ocurridas fueron mínimas, en la orden de centésimo de centímetros, es necesario notar que cualquier alteración en la posición de los dientes puede reflejar dificultad para el ajuste y mantenimiento de la curva de compensación ya que, de acuerdo con Mahler (24), cada movimiento horizontal de 0,01 cm repre- senta un incremento de 1 milímetro en la dimensión vertical.

En el aspecto clínico, el remontaje de las prótesis en el articulador después del procesamiento, es de extrema importancia para que se realice un adecuado ajuste oclusal, principalmente en la región de los dientes posteriores, asegurando así la comodidad y función adecuada de las prótesis, previniendo cualquier efecto nocivo que ésta pueda causar debido a los contactos prematuros que ocurren por la modificación de la oclusión establecida en cera.

\section{CONCLUSIÓN}

Delante de los resultados encontrados en este trabajo, podemos concluir:

1. Todos los grupos estudiados sufrieron alteraciones dimensionales entre el padrón de cera y la prótesis polimerizada.

2. En los grupos donde si utilizó la menor presión (800 kgf), durante la fase de prensado (G1 y S1), ocurrieron las mayores alteraciones en la posición de los dientes artificiales.

3. El grupo de inclusión con yeso y presión de 1.250 kgf; presentó la menor alteración.

4. Los grupos que recibieron presión de $1.000 \mathrm{kgf}$ (yeso y silicona) presentaron valores de alteración muy cercanos.

\section{BIBLIOGRAFÍA}

1. Tucker KM, Freeman BJ. The effect of investing material on processing changes in complete dentures. J Prosthet Dent, 1971;25(Feb. 3): 206-10.

2. Mainieri ET, Boone ME, Potter RH. Tooth movement and dimensional change of denture base materials using two investments methods. J Prosthet Dent, 1980;44(Oct.4):368-73.

3. Cury AAA. Influência do material de revestimento sobre as propriedades físico-químicas das resinas acrílicas polimerizadas com energia de microondas. Piracicaba, 1998. 87p. Tese (Livre 
Gennari Filho H, Coelho Goiato M, Mara Negrão Alves L, Ramos Silva C, Mary Falcón Antenucci R. Evaluación del efecto de prensado en prótesis totales maxilares sobre la movilidad de los dientes artificiales, en función del tipo de inclusión

Docência. Faculdade de Odontologia, Universidade Estadual de Campinas.

4. Zannetti GR. Influência da fase de presangem da resina acrílica e material de inclusão sobre as distâncias entre os dentes em próteses totais polimerizadas por energia de microondas. Piracicaba, 1999. 148p. Dissertação (Mestrado). Faculdade de Odontologia, Universidade Estadual de Campinas.

5. Shibayama R. Analise da variação da posição relativa dos dentes artificiais durante o processamento de próteses totais superiores mensuradas por computação gráfica. Araçatuba, 2002, 127 p. Dissertação de Mestrado, Faculdade de Odontologia de Araçatuba. UNESP.

6. Gennari Filho H, Vedovatto E, Lazari Jab, Assunção WG, Shibayama R. Avaliação comparativa entre três métodos de inclusão de próteses totais polimerizadas pela energia de microondas. Revista Odontológica de Araçatuba, 2003;24(Ag/ set.2):28-34, (A).

7. Gennari Filho H, Vedovatto E, Lazari JAB; Assunção WG, Shibayama, R. Avaliação comparativa da posição relativa dos dentes artificiais entre três métodos de inclusão de próteses totais polimerizadas em banho de água quente. Cienc Odontol Bras. 2003;6(4):32-40, (B).

8. Shibayama R. Analise da variação da posição dos dentes artificiais durante as fases específicas do processamento de próteses totais superiores mensuradas por computação gráfica. Araçatuba, 2006, 148 p. Tese de Doutorado, Faculdade de Odontologia de Araçatuba. UNESP.

9. Gennari Filho H, Martins F, Rocha EP, Assunção WG. Estudo comparativo da inclusão de prótese totais superiores com gesso e barreira de silicone com cobertura total e parcial. Rev ABO Nac 2005;13(2):103-9.

10. Kimura H, Teraoka F, Saito T. Application of microwave for dental technique (Part 2). Adaptability of cured acrylic resins. J Osaka Univ Dent Sch 1984;24(Dec.):21-9.
11. Levin B, Sanders JL, Reitz PV. The use of microwave energy for processing acrylic resins. J Prosthet Dent. 1989; 61(Mar.3):381-3,

12. Sanders JL, Levin B, Reitz PV. Comparison of the adaptation of acrylic resin cured by microwave energy and conventional water bath. Quintessence Int, 1991:22(Mar.3):181-6.

13. Goiato MC, Rahal JS, Gennari Filho H, Fajardo RS, Gonçalves WA. Avaliação da alteração dimensional e porosidade em resinas acrílicas entre métodos de polimerização convencional e por microondas. Rev Fac Odontol Porto Alegre, 2000; 42(Dec.2):37-40.

14. Goiato M, Amantéa DCZ, Vedovatto E, Gennari Filho H, Assunção WG, Santos DM. Estudo comparativo entre duas resinas acrílicas e dois silicones utilizados para o processamento de próteses totais através da análise bidimensional da posição dos dentes artificiais. Cienc Odontol Bras 2005:8(2):60-9.

15. Goiato M, Vedovatto E, Amantéa DCZ, Gennari Filho H, Marinho MLVD. Análise da movimentação dos dentes artificiais em próteses totais superiores. Influência do tipo de polimento. Cienc Odontol Bras, 2006;9(1):6-16.

16. Mazaro JVQ. Influência do processamento de próteses totais maxilares em função da espessura da base. Araçatuba, 2005, 132 P. Dissertação de Mestrado, Faculdade de Odontologia de Araçatuba. UNESP.

17. Vedovatto E. Influência do processamento de próteses totais superiores em função da profundidade do palato: análise da movimentação dos dentes artificiais por computação gráfica. Araçatuba, 2005, 186 p. Dissertação de Mestrado, Faculdade de Odontologia de Araçatuba. UNESP.

18. Assunção WG, Marinho MLVD, Santos PH, Gennari Filho H, Goiato MC. Avaliação da alteração dimensional de silicones para inclusão laboratorial Revista de Odontologia da UNESP. 2006;35(2):113-8. 
19. Russi S, Lombardo JG, Compagnoni MA, Nogueira SS, Mollo Jr, FA. Dimensão Vertical de bases de dentaduras completas. RGO, 1996;4(2): 99-101.

20. Cunha VP, et al. Mufla Bi-maxilar HH-Um recurso laboratorial para oclusão ótima em próteses totais. Rev. Bras. Prótese Clínica \& Laboratorial, 2000;2(8).26-31.

21. Tenguan VLS, et al. Próteses totais confeccionadas pela técnica da zona neutra acrilizadas em muflas HH. Rev. Bras. Prótese Clínica $\mathcal{E}$ Laboratorial, 2001;3(3):183-8.

22. Domitti SS. et al. Alterações oclusais ocorridas na confecção das próteses totais superiores. Rev. Odontol. UNESP, 1998;27(2).427-35.

23. Jamani KD, Moligoda AMA. Effect of denture thichness on tooth movement during processing of complete dentures. Journal of Oral Rehabilitation, 1998;25(9):725-9.
24. Sadamori S, Ganefyanti T, Hamada T, Arima T. Influence of thickness and location on the residual monomer content of denture base cured by three processing methods. J Prosth Dent, 1994; 72(1):19-22.

24. Mahler DB. Inarticulation of complete denture processed by compression molding technique. J Prosthet Dent 1951;1(4):551-9.

\section{CORRESPONDENCIA}

Humberto Gennari Filho

Facultad de Odontología de Araçatuba.

Universidad Estadual Paulista "Julio de Mesquita Filho"

Rua Jose Bonifácio, 1193

CEP: 16015-050

Araçatuba

São Paulo - Brasil

Email: gennari@foa.unesp.br 